\title{
Avaliação do conhecimento dos estudantes de enfermagem sobre sepse
}

Evaluation of the knowledge of nursing students regarding sepsis

Evaluación del conocimiento de los estudiantes de enfermería sobre sepsis

Josilene Fioravanti dos Santos ${ }^{1}$, Amanda Pavinski Alves ${ }^{2}$, Angelita Maria Stabile ${ }^{3}$

\section{RESUMO}

O objetivo deste trabalho foi avaliar o conhecimento dos estudantes do último ano de graduação em Enfermagem, de uma Escola de Enfermagem do interior do estado de São Paulo, sobre a síndrome da resposta inflamatória sistêmica (SIRS), sepse, sepse grave e choque séptico. Foi realizado um estudo descritivo e quantitativo, com a participação de 77 estudantes, no qual se utilizou um instrumento elaborado pelas autoras e validado em seu conteúdo e aparência. Os participantes relataram que possuem pouco conhecimento sobre a temática (58\%) e que o curso de graduação forneceu pouca informação sobre sepse (65\%). Apenas $26 \%, 12 \%, 19 \%$ e $26 \%$ souberam reconhecer quando um paciente encontra-se, respectivamente, com SIRS, sepse, sepse grave e choque séptico. Nossos resultados demonstraram que os estudantes possuem pouco conhecimento sobre sepse, e o pouco conhecimento sobre a temática pode estar relacionado com o ensino deficitário desse assunto no curso de graduação.

Descritores: Educação em Enfermagem; Conhecimento; Sepse; Choque Séptico.

\section{ABSTRACT}

The purpose of this study was to evaluate the knowledge of undergraduate students in the last year of their nursing program in a Nursing School in the interior of the state of São Paulo, regarding systemic inflammatory response syndrome (SIRS), sepsis, severe sepsis and septic shock. A descriptive quantitative study was developed with the participation of 77 students and the use of an instrument elaborated by the authors and validated in terms of its content and appearance. Participants reported they had little knowledge regarding the theme (58\%) and that their nursing program provided little information regarding sepsis (65\%). Only $26 \%, 12 \%, 19 \%$ and $26 \%$ respectively were able to recognize symptoms and signs of SIRS, sepsis, severe sepsis and septic shock. Results demonstrated that students have little knowledge regarding sepsis, and this may be related to a deficiency in the graduate course.

Descriptors: Education, Nursing; Knowledge; Sepsis; Shock, Septic.

\section{RESUMEN}

Se objetivó evaluar el conocimiento de estudiantes del último año de graduación en Enfermería de una Escuela de Enfermería del interior del estado de São Paulo, sobre el síndrome de respuesta inflamatoria sistémica (SIRS), sepsis, sepsis grave y shock séptico. Se realizó estudio descriptivo, cuantitativo, con participación de 77 estudiantes, utilizándose un instrumento elaborado por las autoras, validado en su contenido y apariencia. Los participantes demostraron poseer pocos conocimientos sobre la temática (58\%). Expresaron que el curso de graduación provee de escasa información sobre sepsis (65\%). Apenas $26 \%, 12 \%, 19 \%$ y $26 \%$ supieron reconocer cuándo un paciente se encuentra, respectivamente, con SIRS, sepsis, sepsis grave y shock séptico. Nuestros resultados demuestran que los estudiantes poseen pocos conocimientos sobre sepsis, dicha carencia puede relacionarse con una enseñanza deficitaria al respecto del asunto durante el curso de graduación.

Descriptores: Educación en Enfermería; Conocimiento; Sepsis; Choque Séptico.

\footnotetext{
${ }^{1}$ Enfermeira. Ribeirão Preto, SP, Brasil. E-mail: josilene.santos@usp.br.

2 Enfermeira. Discente do Programa de Pós-Graduação em Enfermagem Fundamental da Escola de Enfermagem da Ribeirão Preto (EERP) da Universidade de São Paulo (USP), nível Mestrado. Ribeirão Preto, SP, Brasil. E-mail: amanda.alves@usp.br.

${ }^{3}$ Enfermeira, Doutora em Fisiologia. Professora Doutora da EERP/USP. Ribeirão Preto, SP, Brasil. E-mail: angelita@eerp.usp.br.
} 


\section{INTRODUÇÃO}

A sepse é um problema de saúde mundial, sendo a principal causa de morte em unidade de terapia intensiva, com taxas variando de 20 a $80 \%$, dependendo da definição utilizada. Anualmente ocorrem, aproximadamente, 18 milhões de casos no mundo(1), sendo que, a cada quatro pessoas diagnosticadas com sepse, uma é por ela vitimada(2). Nos Estados Unidos (EUA), a incidência de sepse está estimada em 751.000 casos a cada ano com taxa de mortalidade de $28,6 \%$, nos pacientes com sepse grave ${ }^{(3)}$.

Estudo Brasileiro de Epidemiologia da Sepse (BASES) mostrou que a taxa de mortalidade dos pacientes com síndrome da resposta inflamatória sistêmica (SIRS), sepse, sepse grave e choque séptico é de $24,2 \%, 33,9 \%, 46,9 \%$ e $52,2 \%$, respectivamente, sendo que a incidência de sepse grave no país é cerca de $27 \%$ em pacientes com mais de 24 horas de internação ${ }^{(4-}$ 5).

Apesar de as altas taxas de mortalidade mostradas nesse estudo serem alarmantes, esses valores podem ser ainda mais altos em decorrência da subnotificação. Um paciente que morre de choque séptico como resultado de pneumonia pode ter a sua causa mortis registrada como pneumonia ao invés de falência múltipla de órgãos, em decorrência de sepse e choque séptico(6).

Dentre as razões para o aumento na incidência de sepse pode-se mencionar o aumento da expectativa de vida, aumento no número de pacientes imuno comprometidos, uso frequente $\mathrm{e}$ indiscriminado de antibióticos e aumento da frequência de procedimentos invasivos ${ }^{(3,7)}$.

Um ponto-chave na redução da mortalidade dos pacientes com sepse e sepse grave é a pronta identificação dos sinais e sintomas e o início precoce do tratamento, como sugere o guideline da Campanha de Sobrevivência à Sepse (Surviving Sepsis Campaing)(8). Entretanto, para o diagnóstico precoce, é necessário que haja um consenso por parte dos profissionais de saúde acerca das definições de SIRS, sepse, sepse grave e choque séptico. Em 1992, as organizações Society of Critical Care Medicine (SCCM) e o American College of Chest Physicians (ACCP) realizaram um Consenso de Sepse, onde propuseram uma uniformização da nomenclatura e uma homogeneização da linguagem usada, de acordo com as manifestações do paciente ${ }^{(8-9)}$.
O enfermeiro é o profissional de saúde mais próximo do paciente, estando em uma posição privilegiada para identificar precocemente os sinais de sepse e prevenir que esta evolua para uma condição ainda mais grave como a sepse grave ou o choque séptico(7,10-11). Para tanto, o enfermeiro deveria possuir o conhecimento necessário para uma avaliação crítica das condições do paciente, detectando a presença de sinais e sintomas sugestivos de sepse e sepse grave e comunicando à equipe médica, para o início do tratamento apropriado. Entretanto, estudos indicam que os profissionais de saúde podem não estar bem informados sobre como identificar corretamente os sinais e sintomas dessas entidades clínicas, ou sobre a importância do tratamento precoce e agressivo na prevenção da deterioração do estado de saúde do paciente ${ }^{(12-13)}$.

Essa deficiência de conhecimento sobre sepse pode ter início ainda no período de formação do futuro enfermeiro. Os conceitos de SIRS, sepse, sepse grave e choque séptico deveriam ser abordados de forma transversal nos cursos de graduação em enfermagem, entretanto, por muitas vezes não fazerem parte da grade curricular, esses conteúdos podem ser negligenciados ou abordados de forma inadequada. A literatura não traz estudos que tenham explorado o conhecimento dos estudantes de enfermagem sobre a sepse, portanto, muitos futuros enfermeiros podem não estar conscientes de sua definição e sinais e sintomas e, como consequência, podem falhar em reconhecer precocemente um paciente séptico.

O objetivo deste trabalho foi avaliar o conhecimento dos estudantes do último ano de graduação em Enfermagem, de uma Escola de Enfermagem do interior do Estado de São Paulo, sobre a síndrome da resposta inflamatória sistêmica (SIRS), sepse, sepse grave e choque séptico. Avaliamos especificamente 0 conhecimento dos estudantes sobre a definição e os sinais e sintomas dessas entidades clínicas.

\section{MÉTODO}

Estudo de delineamento transversal e descritivo de abordagem quantitativa, realizado no período de março a abril de 2011 com os graduandos de uma Escola de Enfermagem de Ribeirão Preto-SP. Para ser incluído no estudo, o estudante deveria estar matriculado no quarto ano do curso de Bacharelado em Enfermagem ou no quinto ano do curso de Bacharelado e Licenciatura da 
referida Escola. Identificamos 113 estudantes que atendiam ao critério de inclusão, destes, 77 concordaram em participar do estudo, representando $68,14 \%$ da população. Esses dois grupos de graduandos encontravam-se no último ano dos seus respectivos cursos de graduação, e a escolha dos mesmos deu-se em função de eles já terem cursado a totalidade das disciplinas que abordaram ou poderiam ter abordado o assunto que é tema deste estudo, em seus conteúdos programáticos.

Para a coleta de dados, utilizou-se um questionário, o qual foi elaborado tendo como referência um questionário validado e utilizado por outros pesquisadores ${ }^{(14)}$ e as experiências das pesquisadoras no assunto. O questionário foi estruturado de forma que todas as questões fossem objetivas e de múltiplas escolhas (alternativas), obedecendo a seguinte ordem: uma questão referente ao conhecimento que os alunos julgavam ter sobre sepse; uma questão sobre as informações que o curso forneceu sobre essa temática; quatro questões apresentadas em forma de estudo de caso para a identificação de SIRS, sepse, sepse grave e choque séptico e duas tabelas de identificação dos sinais/sintomas do paciente séptico e sepse grave, apresentadas em forma de SIM, NÃO e NÃO SEI. Esse questionário foi submetido à validação por um Comitê de Especialistas, composto por três enfermeiras, sendo uma com experiência na validação de escalas; uma pesquisadora da temática sepse; uma com experiência prática em terapia intensiva. Os especialistas avaliaram a aparência e o conteúdo do instrumento, analisando sua objetividade, clareza e pertinência com os propósitos do estudo.

Os estudantes tomaram conhecimento e foram convidados a participar da pesquisa por meio de correio eletrônico. No dia agendado para a coleta de dados, os estudantes foram esclarecidos sobre os objetivos da pesquisa, e aqueles que concordaram em participar, mediante assinatura do Termo de Consentimento Livre e Esclarecido, responderam ao questionário de coleta de dados. Foi solicitado que os estudantes respondessem as perguntas na ordem em que elas apareciam e que não retornassem a nenhuma questão após ler as seguintes.

Para armazenamento dos dados e verificação da frequência da variável investigada no estudo, foi utilizado o programa Excel, com realização de dupla digitação com vistas à verificação de possíveis erros de transcrição.

A execução deste trabalho foi aprovada pelo Comitê de Ética em Pesquisa da Escola de Enfermagem de Ribeirão Preto - USP (Protocolo n¹243/2010). Este trabalho foi desenvolvido de acordo com as diretrizes contidas na Resolução CNS 196/96, para o desenvolvimento de pesquisa com seres humanos.

\section{RESULTADOS}

Dos 77 participantes, 97,3\% eram do sexo feminino e 2,7\%, do sexo masculino. Em relação ao conhecimento que os graduandos julgam ter sobre sepse, seus sinais e sintomas, tratamento e complicações, apenas um (1\%) julga ter nenhum conhecimento; 45 (58\%) julgam ter pouco conhecimento; 29 (38\%) julgam ter um moderado conhecimento; apenas dois (3\%) julgam ter muito conhecimento como demonstrado no Gráfico 1. O mesmo perfil foi observado quando interrogados a respeito das informações que o curso thes forneceu sobre a temática, sendo que seis ( $8 \%$ ) dos participantes relatam nenhuma informação; 50 (65\%), pouca informação; 19 (25\%), moderada informação; e apenas dois (3\%) relatam que o curso forneceu muita informação sobre a sepse (Gráfico 2). 
Gráfico 1: Representação de como os estudantes de enfermagem avaliam o seu conhecimento sobre sepse e seus sinais e sintomas. Ribeirão Preto, SP, 2011.

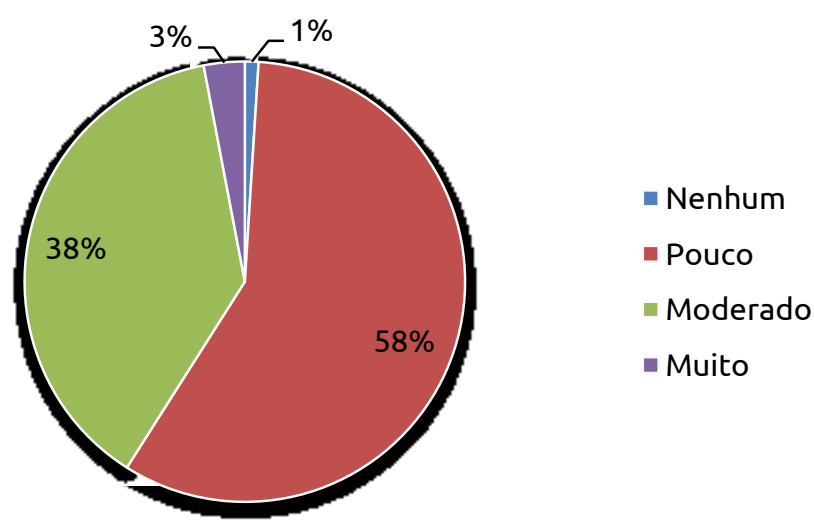

Gráfico 2: Representação da avaliação dos estudantes de enfermagem do quanto o curso de graduação lhes forneceu de informações sobre sepse e seus sinais e sintomas. Ribeirão Preto, SP, 2011.

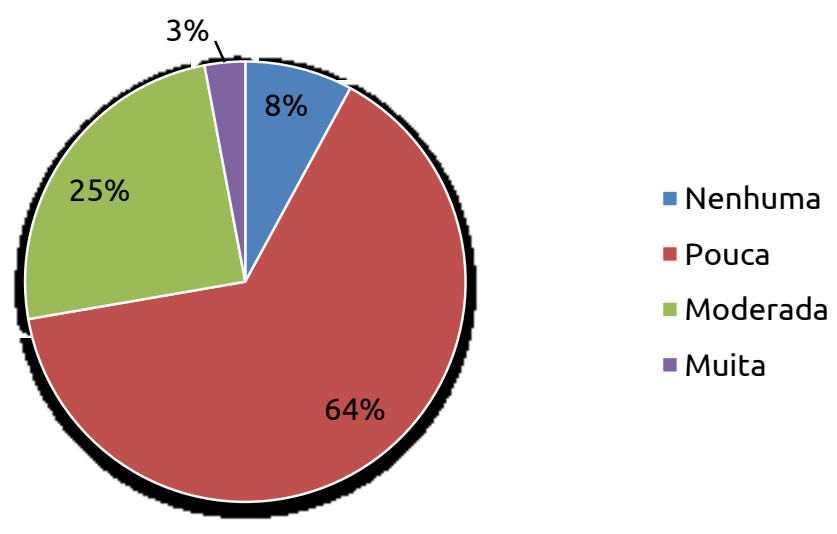

Apenas $26 \%, 12 \%, 19 \%$ e $26 \%$ dos graduandos souberam reconhecer quando um paciente se encontrava em SIRS, sepse, sepse grave e choque séptico, respectivamente (Tabela 1). Ainda, $27 \%, 29 \%$,
$39 \%$ e $19 \%$ dos graduandos relatam não saber diferenciar, classificar e reconhecer os pacientes nessas situações.

Tabela 1: Conhecimento dos estudantes de enfermagem sobre a definição de SIRS, sepse, sepse grave e choque séptico. Ribeirão Preto, SP, 2011.

\begin{tabular}{ccccc}
\hline \multirow{2}{*}{ Definições } & \multicolumn{2}{c}{ Acertos } & \multicolumn{2}{c}{ Não sei } \\
\cline { 2 - 5 } & Frequência & \% & Frequência & \% \\
\hline SIRS & 20 & 26 & 21 & 27 \\
Sepse & 9 & 12 & 30 & 39 \\
Sepse grave & 15 & 19 & 20 & 26 \\
Choque séptico & 20 & 26 & 22 & 29 \\
\hline
\end{tabular}

Quanto a reconhecerem os sintomas da sepse, 72 (94\%) e 66 (87\%) dos graduandos reconhecem que a temperatura corporal superior a $38^{\circ} \mathrm{C}$ é um sintoma sugestivo de sepse e sepse grave. No entanto, apenas quatro (5\%) e sete (9\%) dos graduandos reconheceram que temperatura corporal inferior a $36^{\circ} \mathrm{C}$ também é um sintoma sugestivo de sepse e sepse grave, respectivamente. Dos 77 graduandos, 56 (73\%), 55 (72\%), 66 (86\%) afirmam que a frequência cardíaca superior a 90 bpm, frequência respiratória inferior a 20 mpm e infecção confirmada são sinais sugestivos de que o paciente apresenta sepse (Tabela 2). Ainda 65 (84\%) 
dos graduandos reconhecem a confusão mental como um sintoma sugestivo de que o paciente apresenta sepse grave (Tabela 3).

Tabela 2: Conhecimento dos estudantes de enfermagem sobre os sinais e sintomas sugestivos de sepse. Ribeirão Preto, SP, 2011.

\begin{tabular}{ccccccc}
\hline Sintomas & \multicolumn{2}{c}{ Sim } & \multicolumn{2}{c}{ Não } & \multicolumn{2}{c}{ Não sei } \\
\cline { 2 - 6 } & Frequência & $\%$ & Frequência & \% & Frequência & \% \\
\hline Temperatura corporal $>38^{\circ} \mathrm{C}$ & 72 & 94 & 2 & 3 & 3 & 4 \\
Temperatura corporal $<36^{\circ} \mathrm{C}$ & 4 & 5 & 61 & 80 & 11 & 14 \\
Frequência cardíaca $>90 \mathrm{bpm}$ & 56 & 73 & 9 & 12 & 12 & 16 \\
Frequência respiratória $<20 \mathrm{mpm}$ & 55 & 72 & 6 & 8 & 15 & 20 \\
Infecção confirmada & 66 & 86 & 5 & 6 & 6 & 8 \\
Suspeita de infecção & 22 & 29 & 34 & 45 & 20 & 26 \\
\hline
\end{tabular}

Tabela 3: Conhecimento dos estudantes de enfermagem sobre os sinais e sintomas sugestivos de sepse grave. Ribeirão Preto, SP, 2011.

\begin{tabular}{|c|c|c|c|c|c|c|}
\hline \multirow{2}{*}{ Sintomas } & \multicolumn{2}{|l|}{ Sim } & \multicolumn{2}{|l|}{ Não } & \multicolumn{2}{|l|}{ Não sei } \\
\hline & Frequência & $\%$ & Frequência & $\%$ & Frequência & $\%$ \\
\hline Temperatura corporal $>38^{\circ} \mathrm{C}$ & 66 & 87 & 4 & 5 & 6 & 8 \\
\hline Temperatura corporal $<36^{\circ} \mathrm{C}$ & 7 & 9 & 56 & 73 & 14 & 18 \\
\hline Frequência cardíaca > 90 bpm & 53 & 69 & 7 & 9 & 17 & 22 \\
\hline Frequência respiratória < 20 mpm & 57 & 74 & 4 & 5 & 16 & 21 \\
\hline Infecção confirmada & 67 & 84 & 5 & 6 & 5 & 6 \\
\hline Glicemia capilar > 110 mg/dl * & 43 & 56 & 6 & 8 & 28 & 36 \\
\hline Confusão mental & 65 & 84 & 1 & 1 & 11 & 14 \\
\hline Pressão arterial sistólica < 90 mmHg & 48 & 62 & 6 & 8 & 23 & 30 \\
\hline Saturação de oxigênio < 93\% & 46 & 60 & 4 & 5 & 27 & 35 \\
\hline Volume urinário inferior a $0,5 \mathrm{ml} / \mathrm{h} / \mathrm{Kg}$ & 50 & 65 & 4 & 5 & 23 & 30 \\
\hline
\end{tabular}

*Em paciente sem Diabetes Mellitus

\section{DISCUSSÃO}

Este estudo avaliou o conhecimento de estudantes de enfermagem sobre SIRS, sepse, sepse grave e choque séptico. Nossos resultados demonstram que esses estudantes são capazes de identificar a maioria dos sinais e sintomas relacionados à sepse e sepse grave, entretanto, não estão familiarizados com suas definições e, portanto, podem não estar bem preparados para relacionar os sinais e sintomas com o quadro clínico do paciente e detectar precocemente essa síndrome.

Enfermeiros que atuam nos diferentes cenários hospitalares poderão se deparar com pacientes com sepse. O reconhecimento precoce dessa síndrome possibilita o início rápido do tratamento, diminuindo a probabilidade de o paciente desenvolver o choque séptico e a sequência de eventos que resultam em falência múltipla de órgãos e morte ${ }^{(12)}$.

A resposta inicial do sistema imunológico aos microorganismos invasores é denominada SIRS e tem por objetivo manter a homeostase corporal. A presença de um agente infeccioso é o principal disparador da SIRS, entretanto pode haver causas não infecciosas incluindo queimaduras, trauma ou cirurgias, sendo que o pessoal de enfermagem e o médico devem avaliar se a SIRS é causada por uma infecção ou outro tipo de acometimento(15). Quando a SIRS está associada a um quadro infeccioso suspeito ou confirmado, ela é então denominada sepse ${ }^{(9)}$. Já a sepse grave corresponde à sepse associada à disfunção de órgãos, hipotensão e hipoperfusão tecidual. O choque séptico é caracterizado por hipotensão persistente que não melhora após a reposição volêmica, sendo necessária a administração de agentes vasoativos para manutenção da pressão arterial em níveis compatíveis com a vida(9). Com base nas respostas dos estudantes, observamos que eles tiveram mais facilidade em reconhecer os quadros extremos como a SIRS (31\%) e o choque séptico (31\%) do que definir quando um paciente encontra-se em sepse (15\%) e sepse grave (23\%).

Neste estudo observamos que há um desconhecimento geral de que a hipotermia pode ser um sinal de sepse, uma vez que apenas $5 \%$ dos estudantes 
conseguiram relacionar a temperatura corporal inferior a $36^{\circ} \mathrm{C}$ com o quadro de sepse. Embora a febre seja reconhecida como uma característica importante da sepse, alguns pacientes com infecções graves apresentam hipotermia. Apesar dos efeitos deletérios da febre, ela está relacionada com maior capacidade adaptativa do organismo, auxiliando na eliminação dos patógenos invasores ${ }^{(16)}$, por meio do aumento da produção de anticorpos e da atividade de neutrófilos e macrófagos ${ }^{(17-18)}$. Assim, a incapacidade de desencadear uma resposta febril pode resultar em evolução pior do paciente séptico e, consequentemente, maior taxa de mortalidade ${ }^{(19)}$.

A deficiência de conhecimento da enfermagem sobre sepse já foi observada em outro estudo realizado no Reino Unido, o qual avaliou o conhecimento de enfermeiros que atuam na área hospitalar sobre as definições de sepse e sepse grave e as primeiras intervenções para essas condições. Os pesquisadores concluíram que esses profissionais não estão familiarizados com o conceito de sepse e com as intervenções recomendadas para o tratamento desta síndrome ${ }^{(20)}$.

A pouca familiaridade, com a definição e sinais e os sintomas de sepse, não está restrita à enfermagem. Um estudo brasileiro que avaliou o conhecimento dos médicos sobre sepse concluiu que eles apresentam dificuldade em reconhecer a sepse e sepse grave, provavelmente devido à dificuldade de reconhecer a disfunção de órgãos e a ausência de conhecimento de que a disfunção de órgãos define sepse grave ${ }^{(14)}$. Outro estudo multicêntrico, realizado para avaliar o conhecimento dos médicos sobre sepse concluiu que, apesar da consciência geral sobre a importância e o impacto da sepse, apenas $22 \%$ dos médicos intensivistas citaram corretamente a definição proposta pela ACCP e pela SCCM. Essa divergência, entre a definição proposta e a entendida pelos médicos, reflete a falta de acordo entre as definições usadas por eles, o que, muitas vezes, pode resultar em diagnósticos errôneos ${ }^{(21)}$.

Por outro lado, programas que promovem a educação dos profissionais de saúde sobre sepse mostram-se efetivos. Estudo(22) demonstrou que a implantação de um programa de reconhecimento precoce dos sinais e sintomas de alerta e tratamento rápido para o choque, por meio da educação de todos os prestadores de cuidados de saúde, incluindo enfermeiros, reduziu para $32,6 \%$ a mortalidade nos pacientes com choque séptico. Outra pesquisa(13) demonstrou que a realização de um programa educacional em sepse com duração de oito horas foi capaz de aumentar o conhecimento de enfermeiros sobre o tema. A educação dos enfermeiros sobre sepse também é uma preocupação da enfermagem brasileira. Desde 2005, um projeto pioneiro denominado "Sepse para Enfermeiros (SEPSSE)" vem buscando ensinar o enfermeiro a prevenir e identificar precocemente a sepse $^{(23)}$.

Neste estudo, o conhecimento insatisfatório dessa temática pelos estudantes de enfermagem pode estar diretamente relacionado com o ensino deficitário desse tema no curso de graduação, visto que $65 \%$ dos participantes referem que o curso lhes forneceu pouca informação sobre a sepse, seus sinais e sintomas, tratamento e complicações.

A principal limitação deste estudo é o fato de os resultados representarem a amostra de apenas uma instituição de ensino, estando sujeitos às características próprias do currículo do curso. Seria importante extrapolar esta investigação para outras instituições, a fim de verificar se essa é uma característica dos cursos de Enfermagem em geral.

\section{CONSIDERAÇÕES FINAIS}

Nossos resultados demonstraram que os estudantes do último ano de graduação em Enfermagem possuem pouco conhecimento sobre a temática. O pouco conhecimento dos estudantes sobre sepse pode estar diretamente relacionado com o ensino deficitário desse tema no curso de graduação, evidenciado pelo baixo número de acertos com relação às definições e aos sintomas de SIRS, sepse, sepse grave e choque séptico. Os futuros enfermeiros precisam ter conhecimento da definição de SIRS, sepse, sepse grave e choque séptico para que possam auxiliar no diagnóstico precoce dessas síndromes e prestar o cuidado devido a esses pacientes, por meio da sistematização da assistência do paciente séptico, considerando os diagnósticos de enfermagem, baseando-se na taxonomia elaborada pelo North American Nursing Diagnosis Association- NANDA e aplicando intervenções de enfermagem pertinentes a esses pacientes.

Apesar de a inserção desse tema nos cursos de graduação exigir um empenho por parte das Escolas de 
Enfermagem e dos professores, este esforço pode ser justificado quando consideramos o alto custo financeiro e social para o sistema de saúde e para a sociedade como um todo. Assim, faz-se necessária a criação de estratégias que possibilitem o ensino efetivo do tema,

\section{REFERÊNCIAS}

1. Jaimes F. A literature review of the epidemiology of sepsis in Latin America. Rev Panam Salud Publica. 2005;18(3):163-71. 2. Dellinger RP, Levy MM, Carlet JM, Bion J, Parker MM, Jaeschke R, et al. Surviving Sepsis Campaign: international guidelines for management of severe sepsis and septic shock: 2008. Intensive Care Med. 2008;34(1):17-60.

3. Angus DC, Linde-Zwirble WT, Lidicker J, Clermont G, Carcillo J, Pinsky MR. Epidemiology of severe sepsis in the United States: analysis of incidence, outcome, and associated costs of care. Crit Care Med. 2001;29(7):1303-10.

4. Silva E, Pedro Mde A, Sogayar AC, Mohovic T, Silva CL, Janiszewski $M$, et al. Brazilian Sepsis Epidemiological Study (BASES study). Crit Care. 2004;8(4):R251-60.

5. Sogayar AM, Machado FR, Rea-Neto A, Dornas A, Grion CM, Lobo $S M$, et al. A multicentre, prospective study to evaluate costs of septic patients in Brazilian intensive care units. Pharmacoeconomics. 2008;26(5):425-34.

6. Ahrens T, Tuggle D. Surviving severe sepsis: early recognition and treatment. Crit Care Nurse. 2004;Suppl:2-13.

7. Dellinger RP, Carlet JM, Masur $\mathrm{H}$, Gerlach $\mathrm{H}$, Calandra T, Cohen J, et al. Surviving Sepsis Campaign guidelines for management of severe sepsis and septic shock. Crit Care Med. 2004;32(3):858-73. 8. American College of Chest Physicians/Society of Critical Care Medicine Consensus Conference: definitions for sepsis and organ failure and guidelines for the use of innovative therapies in sepsis. Crit Care Med. 1992;20(6):864-74.

9. Bone RC, Balk RA, Cerra FB, Dellinger RP, Fein AM, Knaus WA, et al. Definitions for sepsis and organ failure and guidelines for the use of innovative therapies in sepsis. The ACCP/SCCM Consensus Conference Committee. American College of Chest Physicians/Society of Critical Care Medicine. Chest. 1992;101(6):1644-55.

10. Kleinpell RM, Graves BT, Ackerman MH. Incidence, pathogenesis, and management of sepsis: an overview. AACN Adv Crit Care. 2006;17(4):385-93.

11. Viana RAPP, organizador. Sepse Para Enfermeiros. São Paulo; 2009.

12. Durthaler JM, Ernst FR, Johnston JA. Managing severe sepsis: a national survey of current practices Am J Health Syst Pharm. 2009;66(1):45-53.

13. Hojatolla Y, Malihe N, Fakhri S. Reviewing the effect of an educational care program in sepsis on knowledge, attitude and practice of nurses in intensive care units in 2011. Iran J Nurs Midwifery Res. 2012;17: S1-6

14. Assunção M, Akamine N, Cardoso GS, Mello PV, Teles JM, Nunes $\mathrm{AL}$, et al. Survey on physicians' knowledge of sepsis: Do they recognize it promptly? J Crit Care. 2010;25(4): 545-52.

15. Dellacroce H. Surviving sepsis: the role of the nurse. RN [Internet]. 2009 [cited $2012 \mathrm{dec}$ 31];72(7):16-21. Available from: http://www.modernmedicine.com/news/surviving-sepsis-role-nurse. 16. Launey $Y$, Nesseler $N$, Mallédant $Y$, Seguin P. Clinical review: fever in septic ICU patients-friend or foe? Crit Care. 2011;15(3):0107.

17. Hasday JD, Singh IS. Fever and the heat shock response: distinct, partially overlapping processes. Cell Stress Chaperones; 2000;5:471480.

18. Sun Z, Andersson R. NF-KB activation and inhibition: a review. Shock; 2002;18:99-106.

19. Trentzsch H, Huber-Wagner S, Hildebrand F, Kanz KG, Faist E, Piltz S, Lefering R; TraumaRegistry DGU. Hypothermia for prediction abordando não só as definições, sinais e sintomas, mas também a sistematização da assistência de enfermagem para os pacientes que se encontram em tais condições, melhorando dessa forma o cuidado de enfermagem.

of death in severely injured blunt trauma patients. Shock; 2012;37(2):131-39.

20. Robson W, Beavis S, Spittle N. An audit of ward nurses' knowledge of sepsis. Nurs Crit Care. 2007;12(2):86-92.

21. Poeze M, Ramsay G, Gerlach H, Rubulotta F, Levy M. An international sepsis survey: a study of doctors' knowledge and perception about sepsis. Crit Care. 2004;8(6):R409-13.

22.Sebat F, Johnson D, Musthafa AA, Watnik M, Moore S, Henry K, et al. A multidisciplinary community hospital program for early and rapid resuscitation of shock in nontrauma patients. Chest. 2005;127(5):1729-43.

23.Compete também à enfermagem detectar e reconhecer a sepse. Coren SP $n^{\circ} 72$ Nov/Dez 2007. (COREN-SP, 2007).

Artigo recebido em 21/07/2011. Aprovado para publicação em 11/06/2012. Artigo publicado em 31/12/2012. 